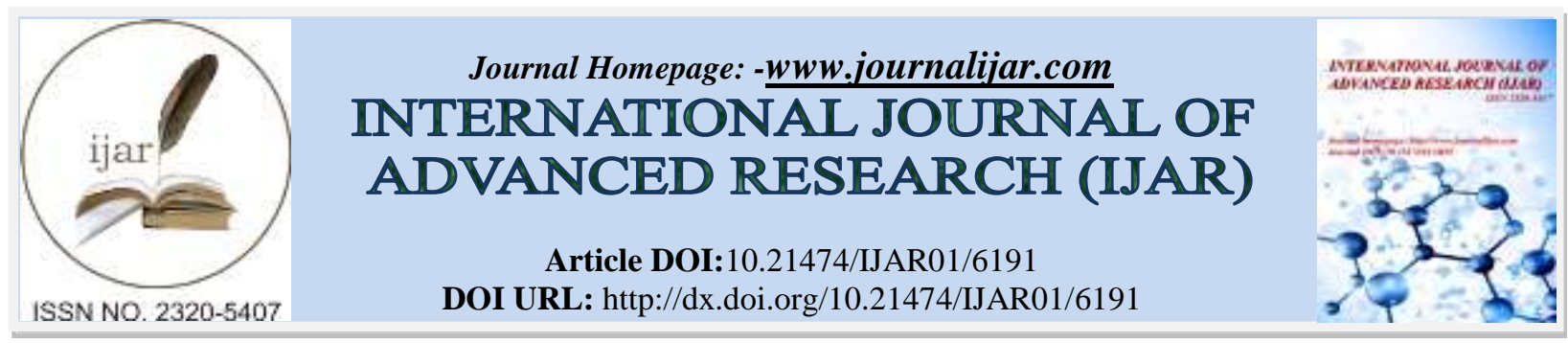

RESEARCH ARTICLE

\title{
BIOSYNTHESIS OF SELENIUM NANOPARTICLES USING ALOE VERA LEAF EXTRACT.
}

"Jay Vyas ${ }^{1}$ and Shafkat Rana ${ }^{2}$.

1. Faculty of science, Pacific Academy of Higher Education and Research University, Udaipur (Rajasthan)INDIA-313003.

2. P.G. Department of Botany, Shri Govind Guru Govt. College, Banswara (Rajasthan)-INDIA-327001.

\section{Manuscript Info}

Manuscript History

Received: 02 November 2017

Final Accepted: 04 December 2017

Published: January 2018

Key words:-

Biosynthesis, Selenium nanoparticles, Aloe vera.

\section{Abstract}

The nanotechnology has recently gained the attention and vast area for research. The biologically synthesis of nanoparticles are eco-friendly, cost effective and non toxic nature. The present study on the reduction of Selenium ions to Selenium nanoparticles mediated through Aloe vera leaf extract were demonstrated with an experimental procedure. The synthesized nanoparticles were characterized using UV-Visible spectrophotometer, Transmission electron microscopy (TEM), Fourier transform spectroscopy (FT-IR) and Energy dispersive X-Ray spectroscopy (EDAX). The selenium nanoparticles synthesized by Aloe vera leaf extract observed as hollow and spherical particles in size ranging 9-58 $\mathrm{nm}$ which found more stable more than two months.

Copy Right, IJAR, 2018,. All rights reserved.

\section{Introduction:-}

Now days nanotechnology has become one of the promising approaches for innovations that lead to fulfill the human interest because of their unique optical, electronic, catalytic properties, photo responsive[1,2] and biomedical application [3,4]. Rapid synthesis of nano scale materials using techniques like laser ablation, pyrolysis, lithography, chemical vapour deposition, sol-gel and electro deposition is very expensive and hazardous. Eco friendly synthesis of nanoparticles using microbes, algae and plant materials are of great interest in the current research. Rapid biosynthesis of nanomaterials using plant materials has more advantages over other biological methods because it is inexpensive and does not need any special conditions [5].

Plants provides a better platform for nanoparticles synthesis as they are free from toxic chemicals as well as provide natural capping agents. More over use of plant extract also reduces the cost of microorganisms isolation and culture media enhancing the cost competitive fesibility over nanoparticles synthesis by microorganisms [6].

Selenium is widely synthesized by chemical reduction method [7]. The synthesis of selenium nanoparticles using biological system in an eco friendly manner. There are few reports on the synthesis of nanoparticles using biological systems. They are Bacillus cereus [8], Klesibella pneumonia [9], Aspergillus terrus [10], leaves of lemon [11], Terminellia arjuna [12], rasin extract of grapes [13] and Bouganvillea spectabilis [14] etc. using for synthesis of nanoparticles synthesis.

In the present research work, we report the synthesis of selenium nanoparticles using Aloe vera extract as a reducing agent. Selenium nanoparticles are formed by reducing the selenium ions present in the solution of sodium selenite 
by the aqueous extract of Aloe vera and characterized using UV-Visible spectrophotometer, Transmission electron microscopy (TEM), Fourier transform spectroscopy (FT-IR) and Energy dispersive X-Ray spectroscopy (EDAX).

\section{Materials and Methods:-}

Preparation of Aloe vera extract

A $30 \mathrm{gm}$ portion of thoroughly washed Aloe vera leaves. Finely cut into $20 \mathrm{ml}$ of sterile distilled water. The extract was then filtered through Whatman filter paper no. 1 and stored at $4{ }^{\circ} \mathrm{C}$ for further work.

\section{Synthesis of metal nanoparticles:-}

Flask containing $25 \mathrm{ml} 5 \mathrm{mM} \mathrm{Na} 2 \mathrm{SeO} 3$ solutions was kept on magnetic stirrer. Then dropwise addition of Aloe vera extract was made in flask containing $\mathrm{Na} 2 \mathrm{SeO} 3$ solution until color of sodium selenite solution changed. From this solution $5 \mathrm{ml}$ was taken which was used as a control. Remaining $20 \mathrm{ml}$ solution was kept in shaker in dark for 72 hrs. After few days the color change of the solution was observed .

\section{UV-Vis spectra analysis:-}

The reduction of metallic selenium ions was observed by measuring the UV-Vis spectrum after 10 to 15 min of color change. A small aliquot was drawn from the solution and a wavelength from $250 \mathrm{~nm}$ to $700 \mathrm{~nm}$ on UV-Vis spectrophotometer (Optizon Double beam 3220) .

\section{TEM analysis:-}

Transmission Electron Microscopic (TEM) analysis was performed with Techni 20 (Philips, Holland). A thin film of the sample was prepared on a carbon coated copper grid by dropping a very small amount of the sample on the grid. The Aloe vera extract containing Se nanoparticles were subjected to centrifugation at $13000 \mathrm{rpm}$ for $10 \mathrm{~min}$. The pellet thus recovered was subjected to washing by its re-suspension in de-ionized water followed by centrifugation at $13000 \mathrm{rpm}$ for $10 \mathrm{~min}$, to remove possible organic contamination present in nanoparticles. Finally, pellet was freeze dried using a lyophilizer (Labconco, Kanas, USA).

\section{EDAX analysis:-}

EDAX analysis was carried out on EDAX XL-30 operating at 15-25KeV.Incorporation of selenium nanoparticles in gauze cloth. Nanoparticles suspension was poured on the gauze cloth discs (diameter $1 \mathrm{~cm}$ ) and there discs were dried at $36^{\circ} \mathrm{c}$ for 7 days.

\section{Sample preparation for Fourier Transform Spectroscopy (FTIR):-}

Metal containing Aloe vera extract for Fourier Transform Infrared (FT-IR) analysis was prepared by mixing $5 \mathrm{mg}$ metal salt in $10 \mathrm{ml}$ plant extract. This metal containing plant extract was incubated at room temperature for 1 hour. After 1 hour incubation, this metal containing leaf extract was dried in Petri plate. After drying, particles were scraped using blade. So, powder of synthesized nanoparticles was obtained. Then spectral scan analysis was carried out at wave number ranging from $400-4000 \mathrm{~cm}-1$ by using a FT-IR spectrometer (Perkin Elmer, Spectrum GX) with resolution of $0.15 \mathrm{~cm}-1$ to evaluate functional groups that might be involved in sorption process.

\section{Results:-}

Visual observation:-

Reduction of metal salts into metal nanoparticles by the bio molecules is always accompanied by the color change of reaction medium. In the present study the colorless solution of sodium selenite is changed in light pink color after drop wise addition of Aloe vera leaf extract at zero hour. As the reduction proceed, the color of reaction medium is gradually changed to dark pink color after 24 hours. 


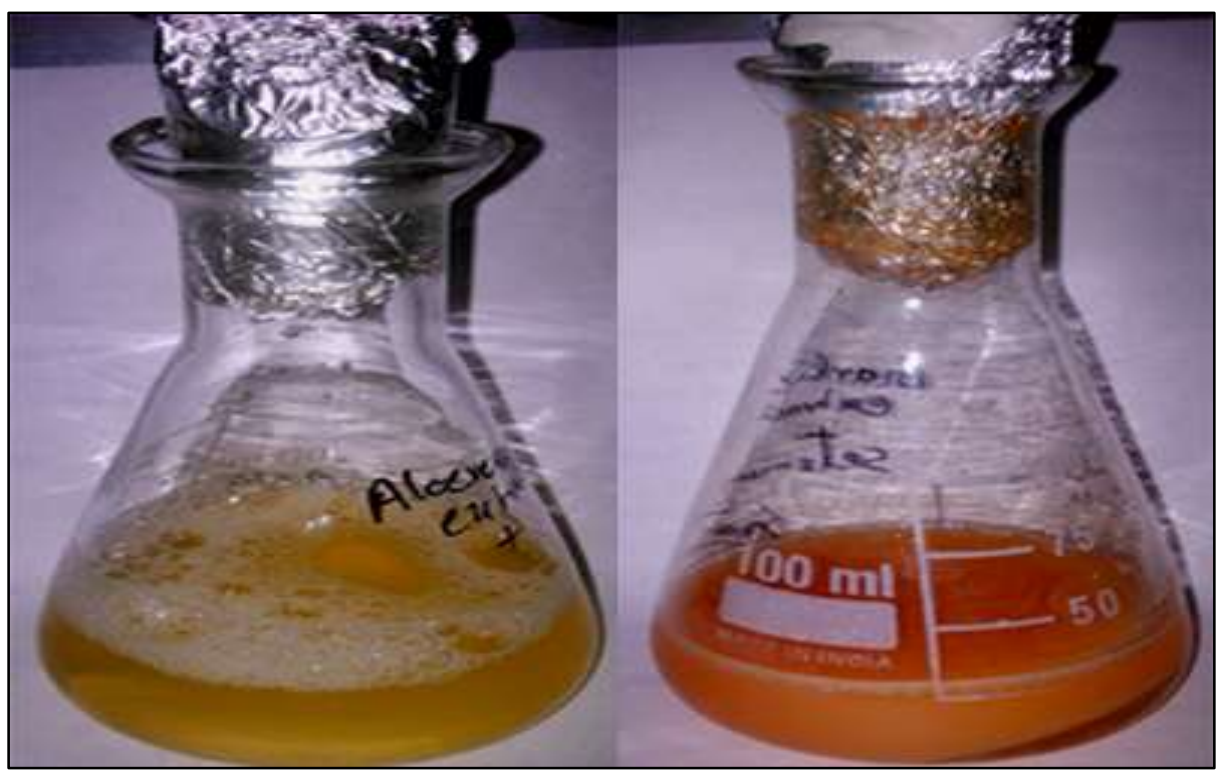

UV - Visible Spectroscopy:-

Fig. 1:-Colour changed after reduction

In order to determine the formation of Selenium nanoparticles in the extract of Aloe vera, a spectral scanning procedure was carried out from $250 \mathrm{~nm}$ to $700 \mathrm{~nm}$. Colloidal solution exhibited absorption maxima at $350 \mathrm{~nm}$ (Fig. 2).

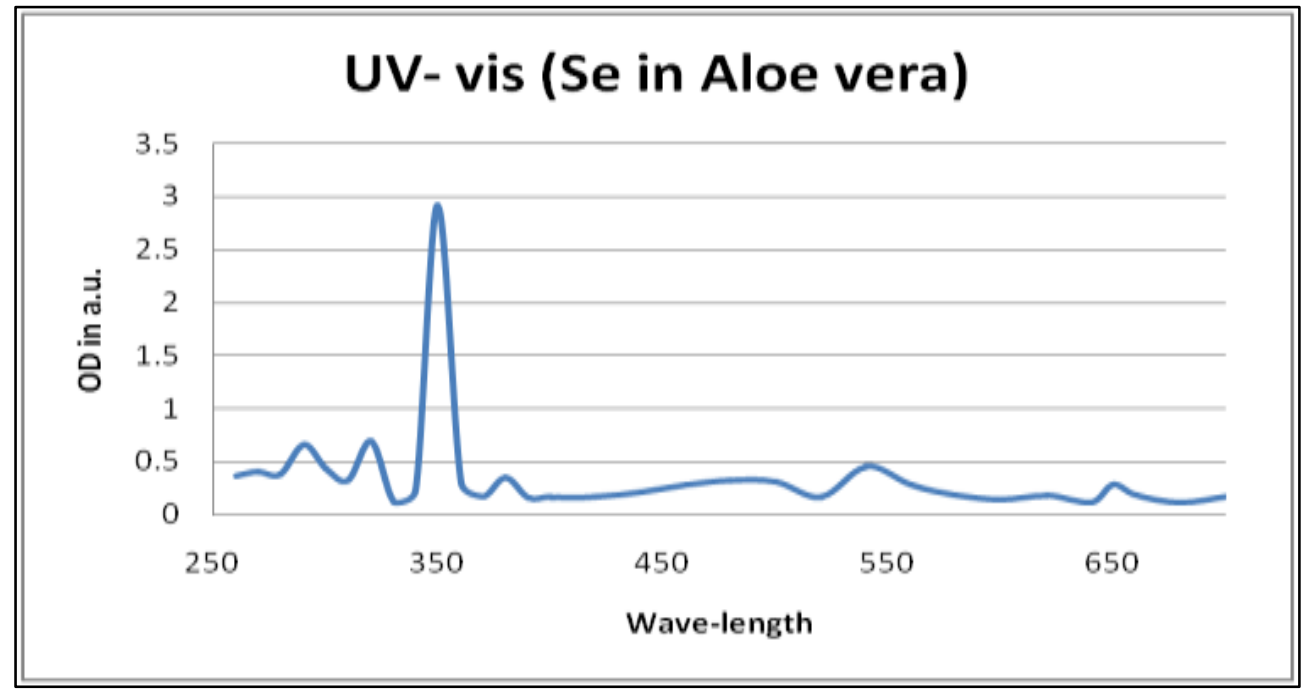

Fig. 2:-UV- VIS spectra selenium nanoparticles

Initially the colloidal solution appeared white in color but after incubation of a period of 24 hours, it turned to reddish brown in color. Building of absorbing maximum at $350 \mathrm{~nm}$ clearly indicates the gradual formation of particles during the incubation period.

\section{Transmission Electron Microscopy (TEM):-}

TEM analysis of colloidal solution indicated the formation of selenium nanoparticles. (Fig.3) shows that size of particles, generated using Aloe vera extract ranges from $9-58 \mathrm{~nm}$. Formation of variable size of particles indicates that particles suggest that Aloe vera extract could form poly disperse nanoparticles. Fig.3 shows Selected Area Electron Diffraction (SAED) of selenium nanoparticles. 


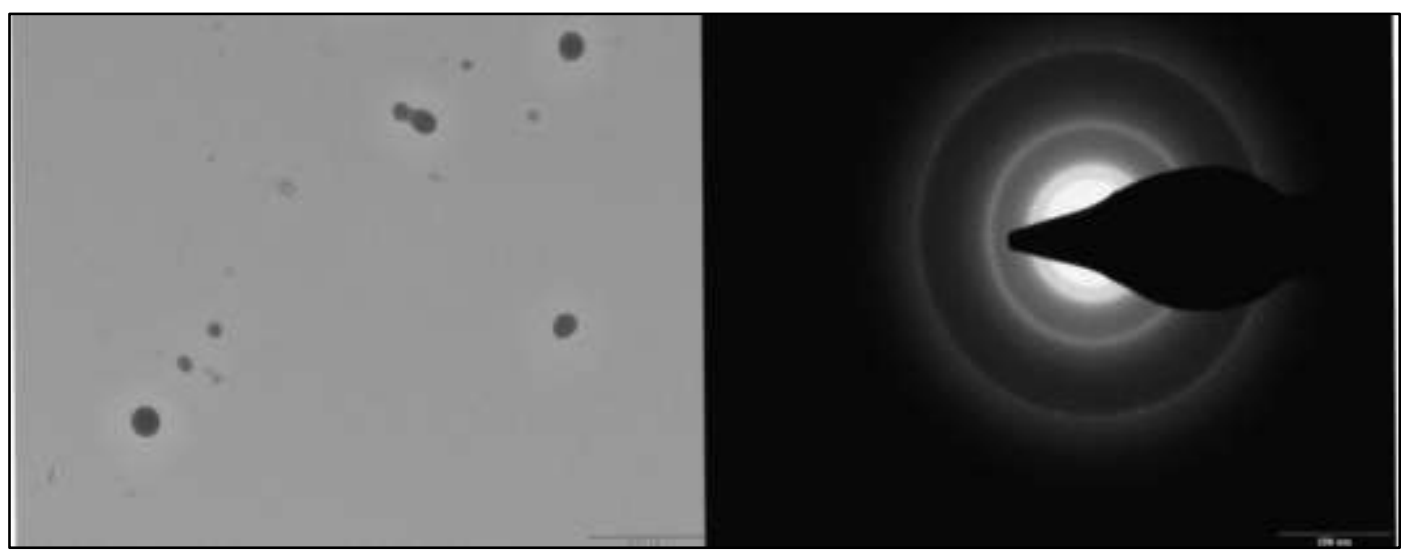

Fig. 3:-TEM analysis of Selenium nanoparticles reveled size of particles 9-58 $\mathrm{nm}$.

Results shows that particles are crystalline in nature as diffraction ring appeared which correspond to diffraction angle of $(111,121$ and 311).

\section{Fourier Transform Infrared Spectroscopy (FT-IR):-}

FT-IR analysis was carried out to identify the possible bio molecules and plant extract-metal ions interaction responsible for formation and stabilization of selenium nanoparticles. The result of FT-IR analysis of plant extract is presented in figure 4 . The figure 4 shows the spectrum of both the sample control (A) and test (B). Fig. 4 (B) shows the spectrum of the sample that contains selenium metal in Aloe vera extract or Fig. 4 (A) shows the spectrum of the Aloe vera extract that did not contain metal selenium. Spectra A show the peaks at 3439.34, 2926.57, 2856.55, $1630.80,1412.22,1384.92,1315.09,1112.49,779.36,618.64$ and $522.63 \mathrm{~cm}-1$. Similarly the transmission peaks of the sample (Fig. 4 B) that did not contain metal selenium were obtained at 3421.21, 2925.59, 2856.55, 1740.26, $1610.60,1415.00,1319.54,1250.14,1077.04,1033.46,812.77,775.97,675.86,599.98$ and $540.99 \mathrm{~cm}-1$.
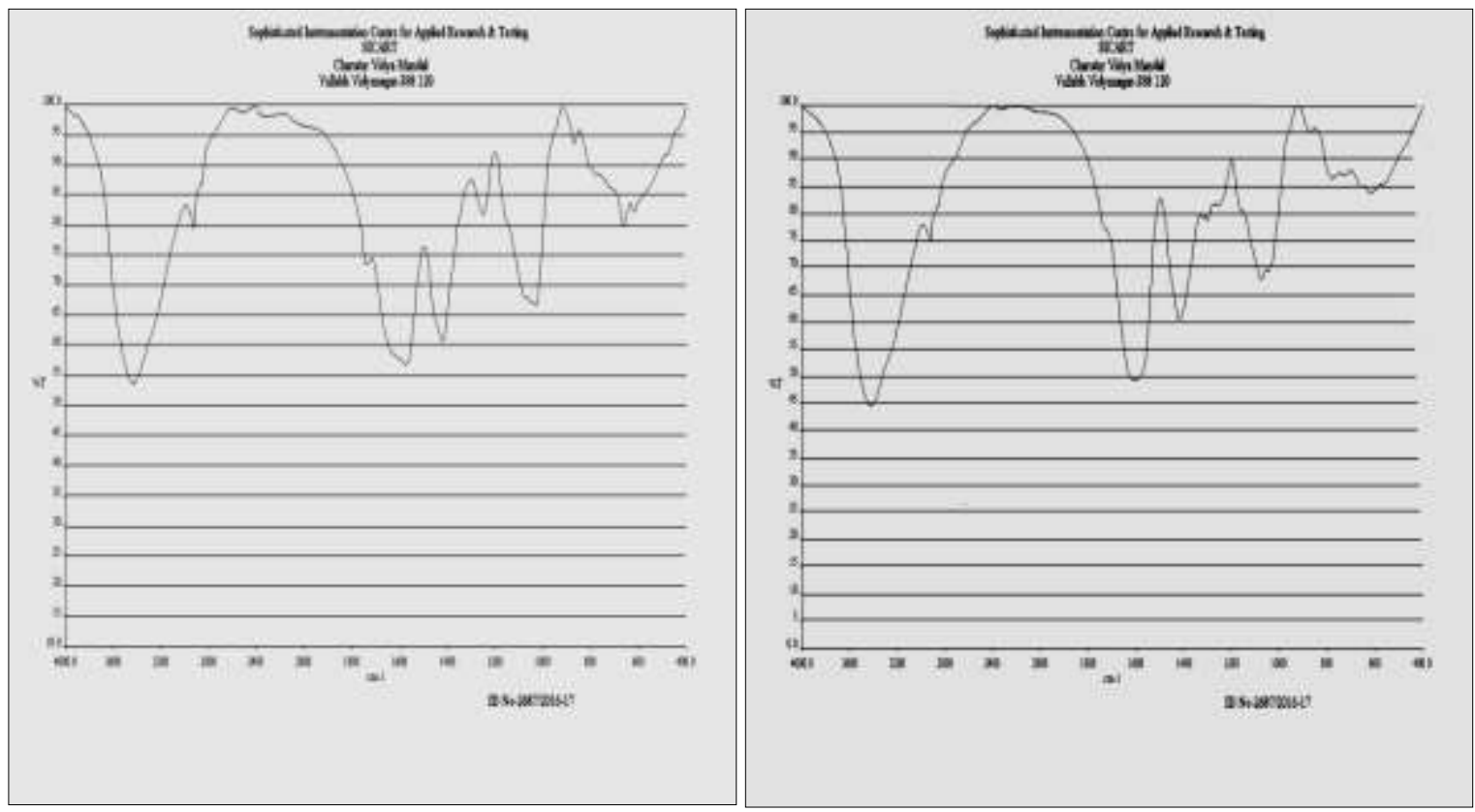

Fig. 4:-FTIR spectrum of (A) Aloe vera extract and (B) Selenium nanoparticles synthesized by Aloe vera extract

Two absorption peaks located around 3400 and 4000 can be assigned as the absorption peak of $\mathrm{N}-\mathrm{H}$. The peaks located around 3000 and 3200 may be due to the presence of $\mathrm{C}-\mathrm{H}$ group. The absorption peaks around 2300 and 2000 can be assigned as the peaks of $\mathrm{CO}_{2}$. The absorption peaks around 1500 and 1800 can be assigned as the absorption peaks of $\mathrm{C}=\mathrm{O} / \mathrm{C}=\mathrm{N} / \mathrm{C}=\mathrm{C}$. The peaks around 1200 and 1100 were attributed to the stretching vibration 
of carboxyl group $(\mathrm{C}=\mathrm{O})$. The peaks around 1100 and 1000 may be due to the presence of $\mathrm{C}-\mathrm{O}$ group. Two absorption peaks around 600 and 500 may be due to the partial deuteration of amine or carboxyl group.

\section{Energy Dispersive X-Ray Spectroscopy (EDAX):-}

EDAX analysis gives qualitative as well as quantitative status of elements that may be involved in formation of nanoparticles. Figure shows the elemental profile of synthesized nanoparticles using Aloe vera extract. The analysis revealed the highest proportion of Selenium (25\%) in nanoparticles followed by oxygen (20\%), sodium (10\%) P (10\%) S (8\%) etc.

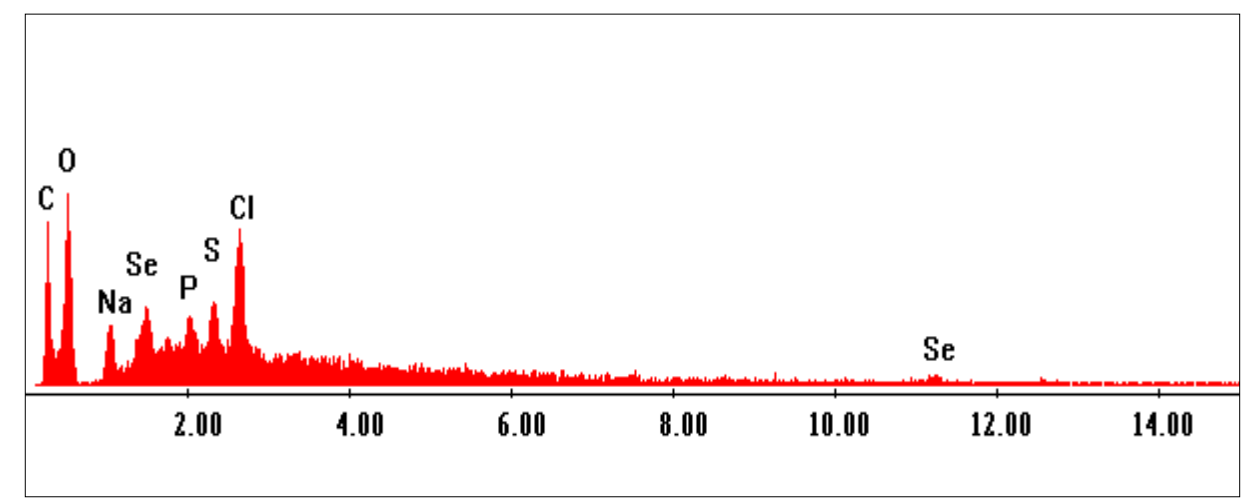

Fig. 5:-EDAX spectrum of selenium nanoparticles

\section{Disscussion:-}

In present work for the biogenic synthesis of selenium nanoparticles Fig.1 represents change in color during nanoparticles synthesis. Initially the colloidal solution appeared colorless but after an incubation period of 24h, it turned to light pink. Building of absorbing maximum at 350nm (Fig.2) clearly indicates the gradual formation of particles during the incubation period. Selenite ions were reduced to selenium nanoparticles after addition of the aqoues Aloe vera extract followed by incubation of the mixture for studied period of time. Reduction of colloidal selenite more over leading to change in color can be attributed to surface plasmone resonance of selenium nanoparticles.

TEM analysis of colloidal solution indicated the formation of selenium nanoparticles. Fig. 3 shows that size of particles generated using Aloe vera extracts ranges $9-58 \mathrm{~nm}$. Formation of variable size of particles suggested that Aloe vera extract could form polydispersed nanoparticles and Fig.3 also shows selected area electron diffraction (SEAD) pattern of selenium nanoparticles. Results indicated that particles were crystalline in nature as diffraction angle of 111,121 and 311 respectively.

FT-IR analysis was carried out to identify the possible formation and stabilization of selenium nanoparticles. The Fig. 4(a) shows the spectrum of the sample that did not contain metal selenium while, Fig. 4(b) shows the spectrum of the sample that contained selenium metal. Spectra 4(a) show peaks at 3415.80, 2934.42, 1634.86, 1415.53, 1049.81 and $607.61 \mathrm{~cm}-1$. Similarly Fig. 4(b) that contained selenium was obtained at 3418.28, 2930.93, 2356.55, 1616.66, 1517.75, 1404.49, 1319.54, 1074.93, 914.94, 816.85, 773.41, 673.81 and $612.46 \mathrm{~cm}-1$. Two absorption peaks located around 3400 and 4000 can be assigned as the absorption peak of $\mathrm{N} \mathrm{H}$. The peaks located around 3000 and 3200 may be due to the presence of $\mathrm{C} \mathrm{H}$ group. The absorption peaks around 2300 and 2000 can be assigned as the peaks of $\mathrm{CO} 2$. The absorption peaks around 1500 and $1800 \mathrm{can}$ be assigned as the absorption peaks of $\mathrm{C} \mathrm{O} / \mathrm{C}$ N/C C. The peaks around 1200 and 1100 were attributed to the stretching vibration of carboxyl group (C O). The peaks around 1100 and 1000 may be due to the presence of $\mathrm{C} \mathrm{O}$ group. Two absorption peaks around 673.81 and 612.46 may be due to the partial deutriation of amine or carboxyl group. The analysis indicated that the selenium nanoparticles formed by Aloe vera extract.

The energy dispersive X-Ray spectroscopy analysis confirmed the presence of elemental selenium nanoparticles in given sample as depiciated by Fig.5. The selenium nanoballs showed characteristics absorption peaks at $1.37 \mathrm{KeV}$ and $11.22 \mathrm{KeV}$. The peak located on the left of the spectrum at around $0.5 \mathrm{KeV}$ clearly indicates the presence of 
oxygen. The lack of other elemental peaks and high amount of selenium in the spectra confirms the purity of the selenium metal in the transformed product.

\section{Conclusion:-}

Present green synthesis shows that the environment friendly Aloe vera can be used as an effective capping as well as reducing agent for the synthesis of selenium nanoparticles. Selenium nanoparticles synthesized by the above method are quite stable and no visible changes are observed even after a month or so, if the nanoparticles solutions are kept in light proof condition. UV-Vis Spectra at $350 \mathrm{~nm}$ with Aloe vera extract and observed as hollow and spherical particles in size ranging $9-58 \mathrm{~nm}$ which is found more stable more than two months. EDAX analysis was carried out to check the presoak of Selenium in nanoparticles. Results of EDAX, confirmed its present. TEM and SEAD represented addition evidence of formation of nanoparticles whereas SEAD indicates the particles were crystalline in nature.

\section{Acknowledgments:-}

The authors are heartly grateful to Dr. Nilanjan Roy, Director, ARIBAS for his support and guidance. I am deeply thankful to Dr. C.L.Patel, CVM, for providing me with state of art facilities and infrastructure which was inevitable for my research work. My sincere thanks to Mr.Vikas and Mr.Sanjay of SICART for their promptness I would also like thank Jaideep Singh Rathore, Director, Wagrshreecollege, banswara (Rajasthan) for constantly motivating me.

\section{Refferences:-}

1. McConnell WP, Novak JP, Brousseau LC, Fuierer RR, Tenent RC, Feldheim DL (2000). Electronic and optical properties of chemically modified metal nanoparticles and molecularly bridged nanoparticle arrays. J Phys Chem B 104:8925-8930.

2. Moreno-Man as M, Pleixats R (2003). Formation of carbon-carbon bonds under catalysis by transition-metal nanoparticles. Acc Chem Res 36:638-643.

3. Lanone S, Boczkowski J (2006). Biomedical applications and potential health risks of nanomaterials: molecular mechanisms. Curr Mol Med 6:651-663.

4. Garnett MC, Kallinteri P (2006). Nanomedicines and nanotoxicology: some physiological principles. Occup Med 56:307-311.

5. CH. Ramamurthy, K. S. Sampath, P. Arunkumar, M. Suresh Kumar, V. Sujatha. K. Premkumar, C. Thirunavukkarasu (2013). Green synthesis and characterization of selenium nanoparticles and its augmented cytotoxicity with doxorubicin on cancer cells. Bioprocess Biosyst Eng 36(8):1131-1139.

6. Garima Singhal, R.B., Kunal Kasariya, Ashish Ranjan Sharma, Rajendra PalSingh (2011). Biosynthesis of silver nanoparticles using Ocimum sanctum (Tulsi) leaf extract and screening its antimicrobial activity. J Nanopart Res 13: 2981-2988.

7. S. Dhanjal, S.S. Cameotra (2010). Aerobic biogenesis of selenium nanospheres by Bacillus cereus isolated from coalmine soil. Microbial Cell Factories 9:52.

8. P.J. Fesharaki1, P. Nazari1, M. Shakibaie, S. Rezaie, M. Banoee, M. Abdollahi, A.R. Shahverdi (2010). Biosynthesis of selenium nanoparticles using Klebsiella pneumoniae and their recovery by a simple sterilization process. Brazilian J. Microbiol 41:461-466.

9. B. Zare, S. Babaie, N. Setayesh, A.R. Shahverdi (2013). Isolation and characterization of a fungus for extracellular synthesis of small selenium nanoparticles. Nanomed. J 1:13 -19.

10. B. Zare, S. Babaie, N. Setayesh, A.R. Shahverdi (2013). Isolation and characterization of a fungus for extracellular synthesis of small selenium nanoparticles. Nanomed. J 1:13 -19.

11. Kumar Suranjit Prasad, Hirnee Patel, Tirtha Patel, Khusbu Patel and Kaliaperumal Selvaraj (2013). Biosynthesis of Se nanoparticles and its effect on UV-induced DNA damage. Colloids and Surfaces B: Biointerfaces 103:261-266.

12. Kumar Suranjit Prasad, Tapan Amin, Saloni Katuva, Madhu Kumari and Kaliaperumal Selvaraj (2014). Synthesis of water soluble CdS nanoparticles and study of their DNA damage activity. Arabian Journal of Chemistry. In Press 2014; Doi j.arabjc.2014.05.033.

13. Garima Sharma, Ashish Ranjan Sharma, Riju Bhavesh, Jongbong Park, Bilguun Ganbold, Ju-Suk Nam and Sang-Soo Lee. Biomolecule-Mediated Synthesis of Selenium Nanoparticles using Dried Vitis vinifera (Raisin) Extract. Molecules 19:2761-2770.

14. Deepa B and Ganesan V. Biogenic Synthesis and Characterization of Selenium Nanoparticles Using the Flower of Bougainvillea spectabilis Willd. International Journal of Science and Research (IJSR) 4:690-695. 
15. Prathna T.C., Lazar Mathew, N. Chandrasekaran, Ashok M. Raichur and Amitava Mukherjee(2010). Biomimetic synthesis of nanoparticles: science, technology and applicability. Biomimetics-Learning from nature 1-20.

16. Mojtaba Shakibaie, Ahmad Reza Shahverdi, Mohammad Ali Faramarzi, Gholam Reza Hassanzadeh, Hamid Reza Rahimi and Omid Sabzevari (2012). Acute and subacute toxicity of novel biogenic selenium nanoparticles in mice. Pharmaceutical Biology 51:58-63.

17. Parisa Jafari Fesharaki, Pardis Nazari, Mojtaba Shakibaie, Sassan Rezaie, Maryam Banoee, Mohammad Abdollahi and Ahmad Reza Shahverdi (2010). Biosynthesis of selenium nanoparticles using Klebsiella pneumonia and their recovery by a simple sterilization process. Brazilian Journal of Microbiology 41:461-466.

18. Kavita K.S., Syed Baker, Rakshith D., Kavitha H.U., Yashwantha Rao H.C., Harini B.P. and Satish S (2013). Plants as Green Source towards Synthesis of Nanoparticles. International Research Journal of Biological Sciences 2:66-7

19. Deendayal Mandal, Mark E. Bolander, Debabrata Mukhopadhyay, Gobinda Sarkar, Priyabrata Mukherjee (2006). The use of microorganisms for the formation of metal nanoparticles and their application. Applied Microbial Biotechnology 69:485-492. 